\title{
Equation of state at finite net-baryon density using Taylor coefficients up to sixth order
}

\author{
Pasi Huovinen ${ }^{\mathrm{a}, \mathrm{b}}$, Péter Petreczky ${ }^{\mathrm{c}}$, Christian Schmidt ${ }^{\mathrm{d}}$ \\ ${ }^{a}$ Institut für Theoretische Physik, Johann Wolfgang Goethe-Universität, 60438 Frankfurt am Main, Germany \\ ${ }^{b}$ Frankfurt Institute for Advanced Studies, 60438 Frankfurt am Main, Germany \\ ${ }^{c}$ Physics Department, Brookhaven National Laboratory, Upton, NY 11973, USA \\ ${ }^{d}$ Fakultät für Physik, Universität Bielefeld, 33615 Bielefeld, Germany
}

\begin{abstract}
We employ the lattice QCD data on Taylor expansion coefficients up to sixth order to construct an equation of state at finite netbaryon density. When we take into account how hadron masses depend on lattice spacing and quark mass, the coefficients evaluated using the $\mathrm{p} 4$ action are equal to those of hadron resonance gas at low temperature. Thus the parametrised equation of state can be smoothly connected to the hadron resonance gas equation of state. We see that the equation of state using Taylor coefficients up to second order is realistic only at low densities, and that at densities corresponding to $s / n_{B} \gtrsim 40$, the expansion converges by the sixth order term.
\end{abstract}

Keywords: lattice QCD, equation of state, hadron resonance gas

One of the methods to extend the lattice QCD calculations to non-zero chemical potential is Taylor expansion of pressure in chemical potentials:

$$
\frac{P}{T^{4}}=\sum_{i j} c_{i j}(T)\left(\frac{\mu_{B}}{T}\right)^{i}\left(\frac{\mu_{S}}{T}\right)^{j} .
$$

The coefficients of this expansion are derivatives of pressure, $P$, with respect to baryon and strangeness chemical potentials, $\mu_{B}$ and $\mu_{S}$, respectively:

$$
c_{i j}(T)=\frac{1}{i ! j !} \frac{T^{i+j}}{T^{4}} \frac{\partial^{i}}{\partial \mu_{B}^{i}} \frac{\partial^{j}}{\partial \mu_{S}^{j}} P\left(T, \mu_{B}=0, \mu_{S}=0\right),
$$

where $T$ is temperature ${ }^{1}$. The lattice QCD calculations of these coefficients have matured to a level where both Budapest-Wuppertal [1] and hotQCD [2] collaborations have published the final continuum extrapolated results for the second order Taylor coefficients, see Fig. 1.

As seen in Fig. 1, at low temperatures the coefficients evaluated using the hadron resonance gas (HRG) model agree with the lattice QCD results. Thus we may expect that HRG is an acceptable approximation of the physical equation of state also at finite net-baryon densities. To check how soon one may truncate the expansion in Eq. (1), we calculate the Taylor coefficients in HRG up to sixth order, evaluate the pressure using up to second, fourth or

\footnotetext{
${ }^{1}$ We use natural units where $c=\hbar=k_{\mathrm{B}}=1$ throughout the text.
} 


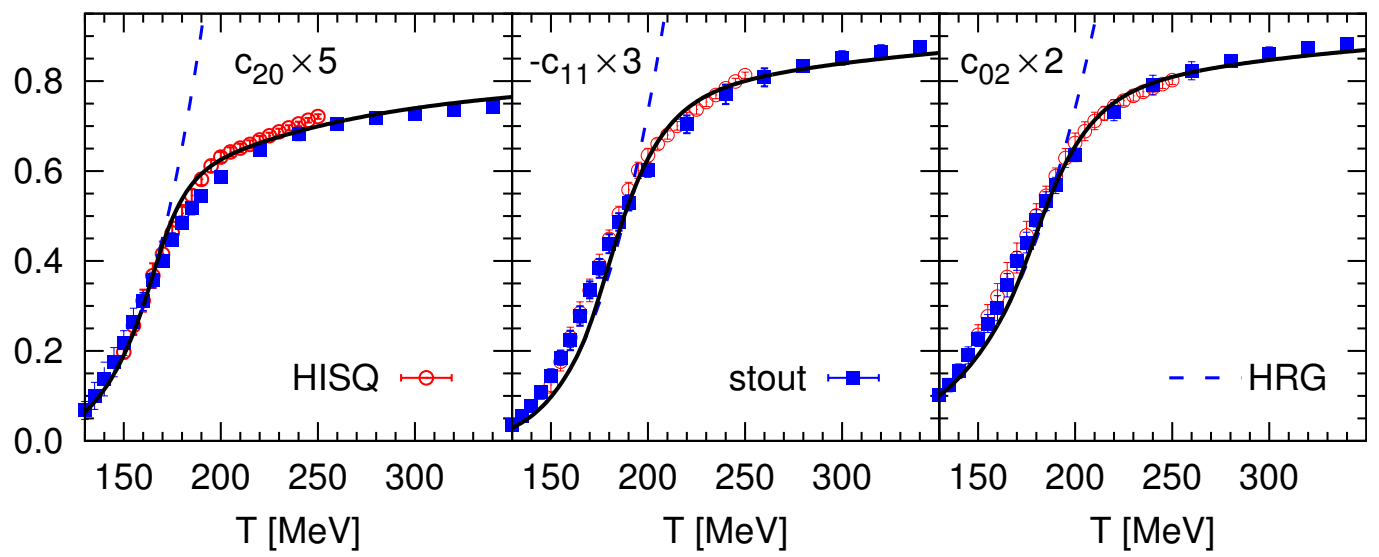

Figure 1. The parametrised (solid line) second order Fourier coefficients compared to HRG values (dashed) and the continuum extrapolated HISQ [2] and stout [1] data.

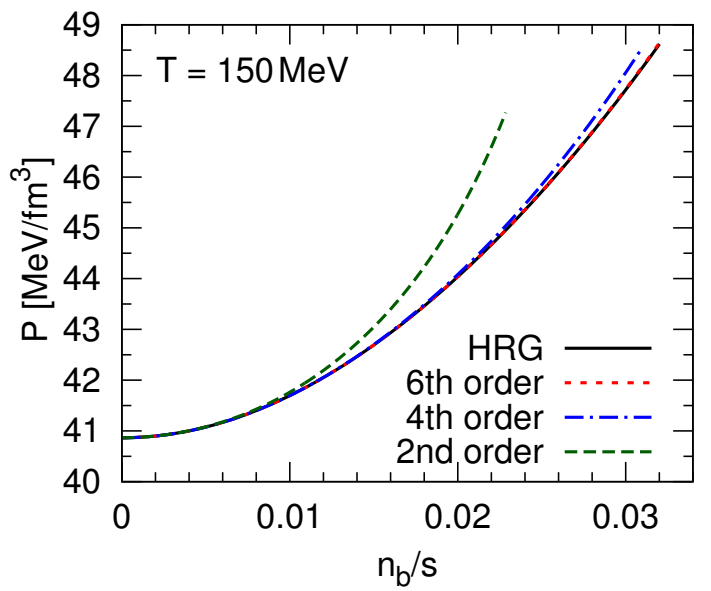

Figure 2. Pressure at constant temperature $T=150 \mathrm{MeV}$ in hadron resonance gas (HRG) and using Taylor coefficients of HRG up to second, fourth and sixth order in Eq. (1).

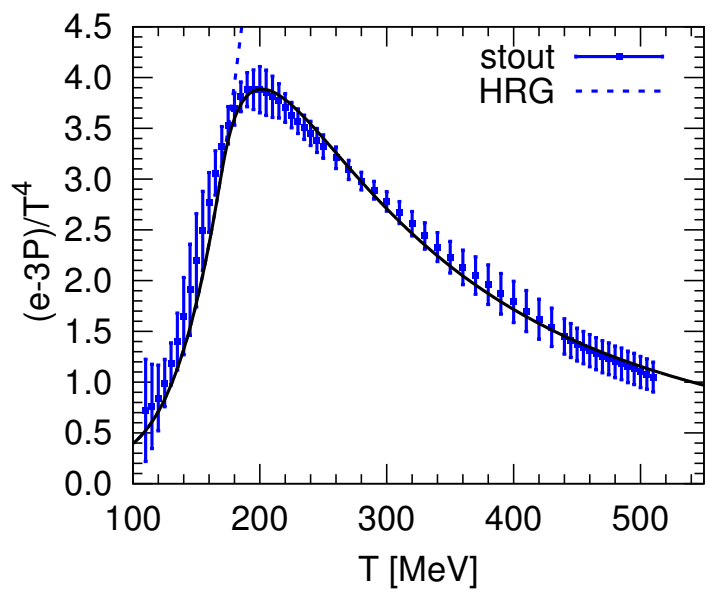

Figure 3. The fitted trace anomaly (solid curve) compared to the HRG trace anomaly (dotted) and the continuum extrapolated lattice QCD result using stout action [11].

sixth order Taylor coefficients, and compare to the actual HRG pressure. The result is shown in Fig. 2, where the pressure at constant $T=150 \mathrm{MeV}$ temperature is shown as a function of inverse of entropy per baryon, $n_{B} / s$. We use $n_{B} / s$ as variable to facilitate easy comparison to heavy-ion collisions at various energies since, unlike net-baryon density $n_{B}$, or baryon chemical potential $\mu_{B}, s / n_{B}$ is (approximately) constant during the entire expansion stage of the collision. We remind that at midrapidity the relevant entropy per baryon is $s / n_{B}=400,100,65$, and 40 at collision energies $\sqrt{s_{N N}} \approx 200,64,39$, and $17 \mathrm{GeV} / \mathrm{fm}^{3}$, respectively. Thus an equation of state based on second order Taylor coefficients only [3] can be expected to be realistic only at relatively low net-baryon densities, $s / n_{B} \gtrsim 100$, i.e., at collisions with collision energy $\sqrt{s_{N N}} \gtrsim 64 \mathrm{GeV}$.

The Taylor coefficients have been evaluated on lattice up to sixth order [4, 5], but unfortunately the fourth and sixth order coefficients suffer from large discretisation errors. As we have argued previously [6, 7, 8, 9], these errors are mostly due to the lattice discretisation effects on hadron masses: When the hadron mass spectrum is modified accordingly (for details see [10]), the HRG model reproduces the lattice data, see Fig. 1 of Ref. [8]. Interestingly this change can be accounted for by shifting the modified HRG result of purely baryonic coefficients towards lower temperature by $30 \mathrm{MeV}[8,9]$. Based on this finding, and because the lattice data agree so well with the modified HRG, we suggest that cutoff effects can be accounted for by shifting the p4 lattice data by $30 \mathrm{MeV}$. The fourth and 


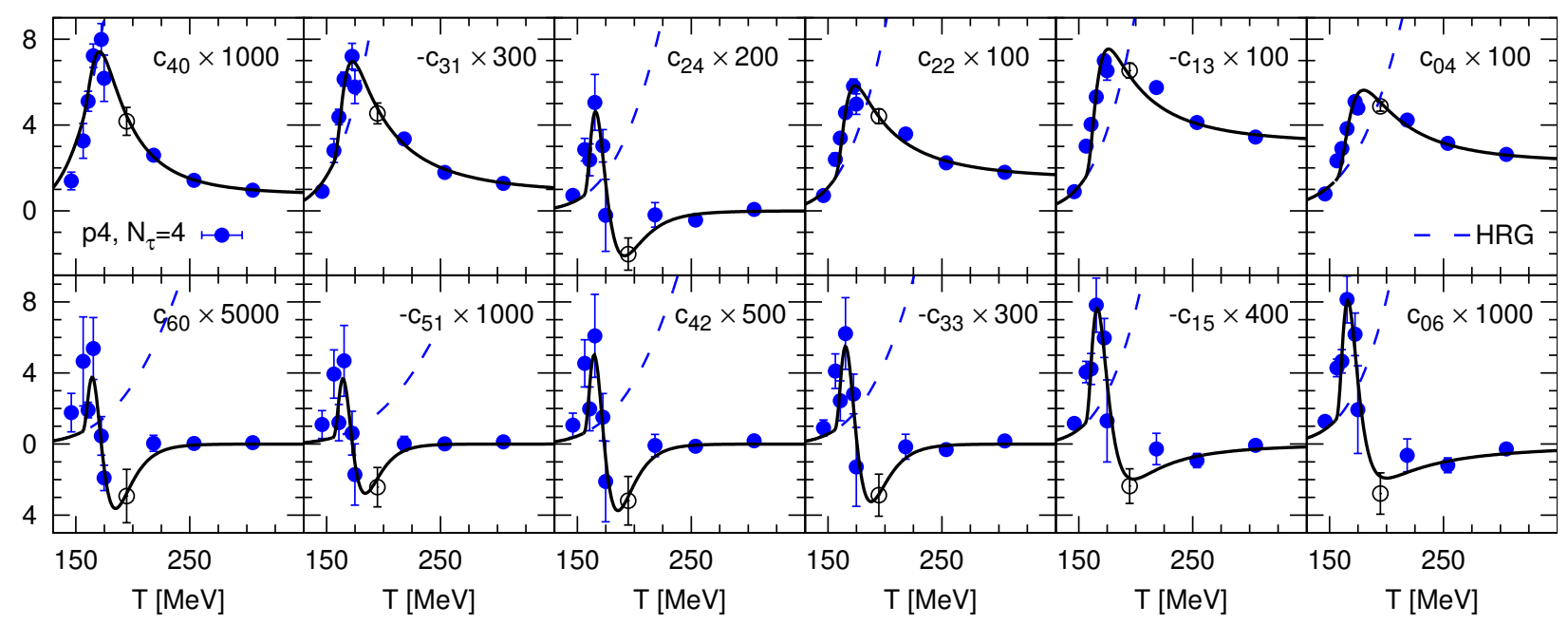

Figure 4. The parametrised (solid line) fourth and sixth order Fourier coefficients compared to HRG values (dashed line) and the shifted p4 data $[4,5]$ (see the text).

sixth order coefficients are shown in Fig. 4, where the data below $206 \mathrm{MeV}$ has been shifted by $30 \mathrm{MeV}$, the data point at $209 \mathrm{MeV}$ by $15 \mathrm{MeV}$ (open symbol), and the points above $209 \mathrm{MeV}$ have not been shifted. At low temperatures the shifted data now agrees with the unmodified HRG.

We parametrise the shifted p4 data, and the unshifted, continuum extrapolated stout and HISQ data, using an inverse polynomial of five (second order) or six (fourth and sixth order coefficients) terms:

$$
c_{i j}(T)=\sum_{k=1}^{m} \frac{a_{k i j}}{\hat{T}^{n_{k i j}}}+c_{i j}^{\mathrm{SB}},
$$

where $c_{i j}^{\mathrm{SB}}$ is the Stefan-Boltzmann value of the particular coefficient, $a_{k i j}$ are the parameters, and the powers $n_{k i j}$ are required to be integers between 1 and 23. $\hat{T}=\left(T-T_{s}\right) / R$ with scaling factors $T_{s}=0$ and $R=0.15 \mathrm{GeV}$ for the second order coefficients and $T_{s}=0.1 \mathrm{GeV}$ and $R=0.05 \mathrm{GeV}$ for all other coefficients. We match the parametrisation of second order coefficients to the HRG value at temperature $T_{\mathrm{SW}}=160 \mathrm{MeV}$, and the fourth and sixth order coefficients at $T_{\mathrm{SW}}=155 \mathrm{MeV}$ by requiring that the Taylor coefficient and its first, second, and third derivatives are continuous. The switching temperatures have been chosen to optimise the fit and lead to smooth behaviour of the speed of sound (see Fig. 5). These constraints fix four (or five) of the parameters $a_{k i j}$. The remaining parameters are fixed by a $\chi^{2}$ fit to the lattice data. The resulting parametrizations are shown as solid curves in Figs. 1 and 4.

We obtain the pressure at $\mu_{B}=0$, i.e. the coefficient $c_{00}$, from the continuum extrapolated stout data for the trace anomaly $(\epsilon-3 P) / T^{4}$ [11], which agree with the very recent HISQ data [12] within errors. As in our earlier parametrisation of trace anomaly [7], we fit the lattice result using an inverse polynomial with four terms, and connect it to the HRG trace anomaly at $T_{\mathrm{SW}}=167 \mathrm{MeV}$ temperature, see Fig. 3.

We characterise the equation of state in Fig. 5a by showing the square of the speed of sound, $c_{s}^{2}=\partial P /\left.\partial \epsilon\right|_{s / n_{B}}$, on various isentropic curves with constant entropy per baryon. The curves at $s / n_{B}=400,65$, and 40 are relevant at collision energies $\sqrt{s_{\mathrm{NN}}}=200,39$ and $17 \mathrm{GeV}$, respectively. At $s / n_{B}=400$ (dotted line), the equation of state is basically identical to the equation of state at $\mu_{B}=0$ (thin solid line). At larger baryon densities the effect of finite baryon density is no longer negligible. The larger the density, the stiffer the equation of state above, and softer below the transition temperature. We see some ripples forming in the transition region with decreasing $s / n_{B}$. These ripples grow fast with increasing density when one goes beyond $s / n_{B}=40$, and therefore we consider $s / n_{B}=40$ to give a practical maximum density for our parametrisation.

On Fig. 5b we have evaluated the square of the speed of sound along the isentropic $s / n_{B}=40$ curve using Taylor coefficients up to second, fourth and sixth order. At temperatures above the transition region they all lead to very 


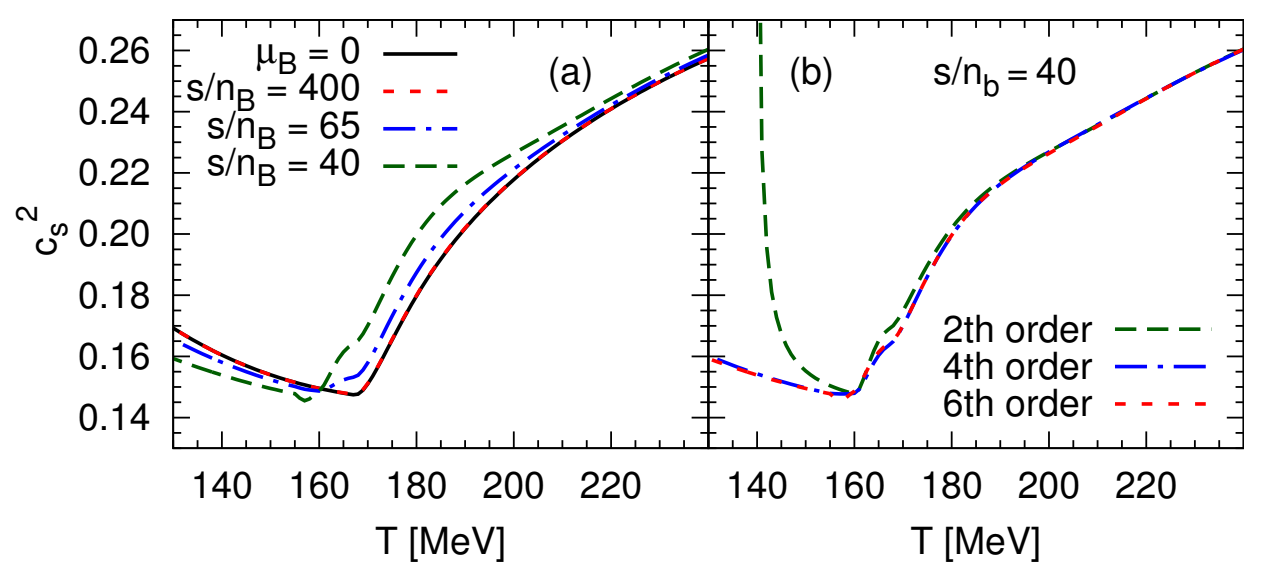

Figure 5. The square of the speed of sound, $c_{s}^{2}$, as a function of temperature on various isentropic curves with constant entropy per baryon (a), and on $s / n_{B}=40$ curve evaluated using Fourier coefficients up to second, fourth or sixth order (b).

similar speed of sound, but below $T \approx 150 \mathrm{MeV}$, the second order result deviates strongly from the fourth and sixth order results. This shows that at low temperatures equation of state based on the second order coefficients only is not sufficient. Furthermore, the small difference between the fourth and sixth order equation of state indicates that if $s / n_{B} \gtrsim 40$, the expansion has basically converged by the sixth order term.

To summarise we have argued that an equation of state based on the Taylor expansion up to second order, is realistic only in collisions with larger collision energy than at the RHIC beam energy scan. We argue that the temperature shift of $30 \mathrm{MeV}$ is a good approximation of the discretisation effects in the lattice QCD data obtained using p4 action. We have constructed an equation of state for finite baryon densities based on hadron resonance gas and lattice QCD data. In such an equation of state, the Taylor expansion essentially converges by the sixth order term if $s / n_{B} \gtrsim 40$.

\section{Acknowledgements}

This work was supported by BMBF under contract no. 06FY9092, and by the U.S. Department of Energy under contract DE-AC02-98CH1086.

\section{References}

[1] S. Borsanyi, Z. Fodor, S. D. Katz, S. Krieg, C. Ratti and K. Szabo, JHEP 1201 (2012) 138.

[2] A. Bazavov et al. [HotQCD Collaboration], Phys. Rev. D 86 (2012) 034509.

[3] S. Borsanyi, G. Endrodi, Z. Fodor, S. D. Katz, S. Krieg, C. Ratti and K. K. Szabo, JHEP 1208 (2012) 053.

[4] C. Miao et al. [ RBC-Bielefeld Collaboration ], PoS LATTICE2008 (2008) 172.

[5] M. Cheng et al., Phys. Rev. D 79 (2009) 074505.

[6] P. Petreczky, PoS ConfinementX (2012) 028.

[7] P. Huovinen and P. Petreczky, Nucl. Phys. A 837 (2010) 26.

[8] P. Huovinen and P. Petreczky, J. Phys. G 38 (2011) 124103.

[9] P. Huovinen, P. Petreczky and C. Schmidt, Central Eur. J. Phys. 10 (2012) 1385.

[10] P. Huovinen and P. Petreczky, J. Phys. Conf. Ser. 230, (2010) 012012.

[11] S. Borsanyi, Z. Fodor, C. Hoelbling, S. D. Katz, S. Krieg and K. K. Szabo, Phys. Lett. B 730 (2014) 99.

[12] A. Bazavov et al., arXiv:1407.6387 [hep-lat]. 